\title{
Hair Strengthening Evaluation of Anisotropic Osmolite Solutions (Inositol + Arginine): Cross-Talk between Dermal Papilla Fibroblast and Keratinocytes of the Outer Root Sheath Using a $\mu$ Hair Follicle 3D Model
}

\author{
Gianni Baratto ${ }^{1}$, Elisa Caviola ${ }^{2}$, Marisa Meloni ${ }^{2}$, Nicola Lionetti ${ }^{3, *}$, Adriana Bonfigli ${ }^{4}$, \\ Maurizio Sironi ${ }^{5}\left(\mathbb{D}\right.$, Stefano Pieraccini ${ }^{5}\left(\mathbb{D}\right.$, Marc Oliver ${ }^{6}$, Luisa Coderch ${ }^{6}$ and Luigi Rigano ${ }^{3, *}$ \\ 1 Unifarco SPA, Via Cal Longa 62, 32035 Santa Giustina, Italy; gianni.baratto@unifarco.it \\ 2 Vitroscreen S.r.l., 20145 Milano, Italy; elisa.caviola@vitroscreen.com (E.C.); \\ marisa.meloni@vitroscreen.com (M.M.) \\ 3 Rigano Laboratories, 20125 Milano, Italy \\ 4 Institute of Skin and Product Evaluation (ISPE) srl, Via Bruschetti 1, 20125 Milano, Italy; a.bonfigli@ispe.it \\ 5 Dipartimento di Chimica, Università degli studi di Milano, 20133 Milano, Italy; \\ maurizio.sironi@unimi.it (M.S.); stefano.pieraccini@unimi.it (S.P.) \\ 6 Department of Chemical and Surfactants Technology, Institute of Advanced Chemistry of \\ Catalonia (IQAC-CSIC), 08304 Barcelona, Spain; montqt@cid.csic.es (M.O.); lcnesl@cid.csic.es (L.C.) \\ * Correspondence: lionetti@thecosmetologist.com (N.L.); rigano@thecosmetologist.com (L.R.)
}

Received: 28 June 2018; Accepted: 9 August 2018; Published: 25 September 2018

\begin{abstract}
The hair follicle (HF) is a dynamic "mini-organ" which undergoes bi-continuous cycles of growth, destruction and rest. The molecular mechanisms underlying the HF cycle are complex yet not fully understood. Anyhow, it is clear that the epithelial-mesenchymal interactions, and in particular the cross-talk between dermal papilla fibroblast (DPF) and the keratinocytes of the outer root sheath (ORSK) play a pivotal role. Aim of this study is the evaluation of the biological activity of anisotropic osmolyte solutions on the HF cycle. As reported in recent studies, dermal papilla cells deeply modify their gene expression profile when cultured as monolayers, but their transcriptional pattern can be partially restored when they are cultured as 3-dimensional spheroids. This draws our attention to the discovery that the spatial distribution of cells in the growth medium is fundamental in order to produce a verisimilar model. Therefore, we used the hanging drop technology to produce a scaffold-free micro-tissue model applied to a DPF-ORSK co-culture in order to create a $\mu \mathrm{HF}$ 3-dimensional model. As a result, this system was capable of evaluating the efficacy of the anisotropic osmolyte solutions on the progressive increase of the follicle turnover and 'health'. Moreover, an in silico model was used in order to screen the most promising combination of osmolyte molecules. In vivo objective evaluations were finally carried out on volunteers having hair disorders.
\end{abstract}

Keywords: hair growth; microtissues; osmolytes; in silico measurements

\section{Introduction}

The hair follicle, a skin appendage, is a self-renewing mini-organ presenting several biological functions:

- $\quad$ Protection from external environment.

- $\quad$ Skin repairing during re-epithelization. 
- $\quad$ Dispersion of sweat and sweat gland products (e.g., pheromones).

- Dispersion of sebum.

- $\quad$ Action as touch receptor.

Moreover, the scalp equilibrium has a psychological and social importance in our society, so the study of the follicle physiology is increasingly interesting for the development of more efficient hair-care products. Hair follicles vary considerably in size and shape, depending on their location, but they all have the same basic structure. Rapidly proliferating matrix cells in the hair bulb produce the hair shaft. Its bulk zone, the cortex, is composed of hair-specific intermediate filaments and associated proteins. The dermal papilla, which is composed of specialized fibroblasts located at the base of the follicle, is thought to control the number of matrix cells and thus the size of hair. Normal development and cycling of hair follicles depend on the interaction of the follicular epithelium with the adjacent mesenchymal dermal papilla [1,2]. The dermal papilla induces the hair-follicle formation from the overlying epithelium during the fetal development. At the onset of each new follicular cycle in adults, the dermal papilla interacts with secondary germ cells in the hair-follicle bulge to regenerate the lower follicle [3,4]. The complexity of the epithelial-mesenchymal interactions, which underlie the HF growth and cycling [5,6], makes it difficult to fully reproduce them in vitro. These interactions, and in particular the cross-talk between dermal papilla (DP) fibroblast and the keratinocytes of the outer root sheath (ORSK) play a pivotal role. The availability of a DPF-ORSK co-culture model allows researchers to investigate the mechanisms that regulate the HF cycle and to study the effects of active compounds in pre-clinical research. As reported in one study [7], dermal papilla cells deeply modify their gene expression profile when cultured as monolayers, but the transcriptional pattern can be partially restored when they are cultured as 3D spheroids. This draws the attention to the spatial distribution of cells, which is fundamental to produce a verisimilar model. The use of a spheroidal co-culture of DPF and ORSK is an optimal method, because it does not require a scaffold to create a $\mu \mathrm{HF}$.

\section{Experimental Design}

The first phase of the project included the screening of the best active molecules and their possible combination. The screening was carried out by means of computerized models applied to the study of the water structure, the behavior of the selected molecules and their interaction with the protein moieties of keratin. The best performing systems were then studied using the microtissues (MTs), which represents an in vitro cell culture method in which cells, instead of adhering to a substrate, forming a two-dimensional monolayer, clump together in droplets suspended in the medium. This gives the possibility to form a three-dimensional structure where the spatial, molecular cell-cell and cell-matrix interactions found in vivo are better mimicked. The cells can grow and organize according to their natural physiology and reproduce in vitro (at least partially) the typical morphology and functions of their tissue of origin. The results of this in vitro study were used to assemble some hair treatment formulations and to test them in ex vivo and in vivo tests.

\section{Materials and Methods}

\subsection{In Silico}

A water-osmolyte system was built by placing the osmolytes in random positions into a $50 \AA$ simulation box. Subsequently, the boxes were filled with water. The 3D structure of a helical keratin segment was obtained from a Protein Data Bank (PDB id 3TNU [8]). The protein was inserted in a virtual box (size $170 \times 60 \times 60 \AA$ ) and amounts of osmolyte molecules (arginine, myo-inositol, taurine or their mixture) necessary to finally reach $1 \mathrm{M}$ concentration were added. Also in this case, osmoprotectors were added in random positions into the simulation box prior to water solvation. The simulations were performed in explicit solvent under periodic boundary conditions. The water structure is described by the TIP4P (Transferable Intermolecular Potential 4 Points) model [9]. All the 
systems were neutralized with a proper number of ions $\left(\mathrm{Na}^{+}\right.$or $\left.\mathrm{Cl}^{-}\right)$to ensure electro-neutrality. Electrostatic interactions were described with the particle mesh Ewald algorithm [10], while a $14 \AA$ cutoff was applied to Van der Waals interactions. The simulations were run with the Gromacs 4.5.3 version [11]. All the systems were submitted to a geometry optimization of 10,000 steps employing the steepest descent method. Afterwards, they were equilibrated for 100 ps at constant temperature and volume and for further 100 ps at constant pressure and temperature, in order to equilibrate respectively the temperature and the density of the systems. The production phase of the simulations consisted in $100 \mathrm{~ns}(100,000 \mathrm{ps})$ of molecular dynamics at constant temperature $(310 \mathrm{~K})$ and pressure (1 bar). The system temperature was kept constant with the v-rescale algorithm [12] and the pressure through weak coupling with a barostat using the Berendsen algorithm [13]. The LINCS (LINear Constraint Solver) [14] algorithm was used to constrain all the bonds to their reference length, in order to use a 2 fs time step. The protein was described with the AMBER99SB-ildn [15] force field, while the osmo-protectors were described with the Generalized Amber Force Field [16]. The two force fields are mutually compatible.

\subsection{In Vitro}

Human hair follicle dermal papilla cells (HDPC) and human hair follicle keratinocytes (HHFK) were used to reconstruct $\mu \mathrm{HF}$ micro-tissue, using the hanging drop method into GRAVITYPLUS plates from InSphero (Zurich, Switzerland). The $\mu \mathrm{HF}$ were then transferred into GRAVITYTRAP for cultivation and treatment with Cyclosporin A (reference for hair growth), Myo-inositol or a mix of Myo-inositol:Arginine 1:1.

In order to evaluate the effect of the anisotropic osmolytes solution on the structure and morphology of the reconstructed models, the $\mu \mathrm{HF}$ were harvested at different time points after 24 and $120 \mathrm{~h}$ of treatment and characterized on the basis of the following parameters.

Histo-morphological analysis: performed by Hematoxilin-Eosin (Histoline) staining of $7 \mu \mathrm{m}$ formalin fixed paraffin embedded (FFPE) sections.

Immunostaining was performed on FFPE sections using anti-human citokeratina CK6 (Sigma, St. Louis, MO, USA) primary antibody and DAB (3,3'-Diaminobenzidine) chromogenic detection method (Life-Technologies, Carlsbad, CA, USA) or anti-human COLIV (Santa Cruz Biotechnology, Inc., Dallas, TX, USA) and fluorescent secondary antibody to monitor the presence and the maintenance of type-specific features. All the images were captured using Leica Instruments (DM2500 microscope (Leica, Wetzlar, Germany) or SP2 confocal microscope (Leica, Wetzlar, Germany) and LASX 10.061 software.

\subsection{Ex Vivo}

All the studies were performed with $1 \mathrm{~g}$ tufts from the same lot of Caucasian (Cau) hair. To evaluate the protective effect of the product (hair spray product HSP), different samples were tested:

Non Irradiated and Non Treated (NN): Cau sample without treatment or irradiation.

Irradiated and Non Treated (IN): Cau sample irradiated but not treated.

Non Irradiated and Treated Sample (NT): Cau sample treated (HSP) $24 \mathrm{~h}$ before analysis and not irradiated.

Irradiated sample after Treatment (TI): Cau sample treated $1 \mathrm{~h}$ before irradiation.

Irradiated sample before treatment (IT): Cau sample irradiated immediately after treatment.

\subsubsection{Irradiation}

Irradiation was carried out for $5 \mathrm{~h}$ using a SUNTEST CPS+ (ATLAS Material Testing Technology $\mathrm{GmbH}$, Linsengericht, Germany) at $250 \mathrm{~W} / \mathrm{m}^{2} 24 \mathrm{~h}$ before protein degradation analysis. 


\subsubsection{Treatment Administration}

Treated samples (NT, TI and IT) were impregnated with $650 \mathrm{mg}$ of HSP and evenly distributed. After treatment, or irradiation in TI sample, all the shafts were stored overnight in a temperature and humidity-controlled room $\left(25^{\circ} \mathrm{C} 50 \% \mathrm{RH}\right)$.

\subsubsection{Protein Extraction and Bradford analysis}

A total amount of hair weighing $100 \mathrm{mg}$ from each sample was cut into small pieces and put into $3 \mathrm{~mL}$ vials with $2 \mathrm{~mL}$ of $2 \%$ SDS (sodium dodecyl sulfate) aqueous solution. To remove the HSP, which could have interfered with the protein quantification, four washes were performed replacing $1 \mathrm{~mL}$ of solution with the same amount of clean $2 \%$ SDS after softly shaking the vials. The cleaned samples were sonicated using a Labsonic 1510 device (B. Braun, Melsungen, Germany) for $5 \mathrm{~h}$ and at $45{ }^{\circ} \mathrm{C}$. Hair extracts were then diluted to $0.01 \%$ SDS concentration and the Bradford colorimetric assay was used to quantify the proteins and peptides solubilized. This assay is based on the formation of a complex between the dye, Brilliant Blue $G$ and the proteins in solution, which leads to an increase in absorption at $595 \mathrm{~nm}$ and is proportional to the amount of protein in the solution. Bovine serum albumin (BSA) was used as a standard to calculate the amount of protein.

\subsubsection{Data Treatment}

The mean values and standard deviations (SD) were calculated.

\subsection{In Vivo}

This was an open-label, single-center, non-randomized study [17]. A hair care treatment was assembled, made of shampoo, spray, serum and hair conditioner formulae. The primary objective of the study was to evaluate the strengthening effect on hair of the twice-weekly treatment in mature women with aged hair, measured through pull test and scanning electronic microscope [18,19]. The primary efficacy endpoints were the statistically significant improvements in the hair resistance to traction and in hair diameter [20]. At the end of the test, volunteers were asked to express their opinion on the efficacy of the treatment by filling in a five-point scale questionnaire. The study was conducted in accordance with the Declaration of Helsinki on 30 female volunteers, between 35 and 65 years old, (average age 49 years) having long, aged, breakable, damaged and weak hair. For each subject, the state of hair was checked during the recruitment visit to verify the correspondence of the volunteers to the inclusion criteria of the study. Among the 30 subjects, 15 had colored or style perm hair and/or were users of hair straighteners while 15 had non-colored hair. Subjects were informed of the nature, purpose and risk of the study and gave their written consent before participating in the test. All subjects were instructed to use the treatment twice a week for six weeks. The study was carried out in a temperature and humidity-controlled room. Volunteers were asked to wash their hair $48 \mathrm{~h}$ before each visit and to refrain from the application of any styling products. The evaluation of benefits was made using a clinical and instrumental technique performed at the baseline and after six weeks of treatment.

\subsubsection{Pull Test}

Clinical evaluation of the resistance of hair to traction was performed by applying a constant traction on hair tufts taken from 3 different areas of the scalp:

- Temporal area: located $3 \mathrm{~cm}$ above the back auricle-line;

- Frontal area: located on a median line, $4 \mathrm{~cm}$ from the frontal hairline;

- Occipital area: located on a median longitudinal line, $4 \mathrm{~cm}$ from the back hairline.

Volunteers were asked to brush their hair two hours before each visit. The dermatologist took a cluster of about 40-70 hairs between thumb and forefinger and pulled them gently applying a slow constant traction, moving toward their distal end. In this way, a number of hairs were removed. 
Growing hairs should remain rooted in place while hair at the end of the telogen phase (exogen) should be extracted easily. The hair resistance to traction was evaluated based on the total number of hairs removed from the three areas. The lower the number of hairs removed the higher the hair resistance to traction.

\subsubsection{Diameter of the Hair}

An instrumental evaluation of the hair diameter was performed by Scanning Electron Microscope SEM, using a FEI Quanta 200 ESEM microscope (Thermo Fisher Scientific, Hillsboro, OR, USA). At each check, some hairs were taken from the apical area of the volunteers' scalp. The hairs were then analyzed through the scanning electronic microscope to measure their diameter (in $\mu \mathrm{m}$ ).

\subsubsection{Subjective Evaluation}

After six weeks of treatment, the volunteers expressed their opinion on the performance of the treatment by filling in a five-point scale questionnaire. The percentage of answers given for each level of intensity was calculated. "All subjects gave their informed consent for inclusion before they participated in the study. The study was conducted in accordance with the Declaration of Helsinki, and the protocol was approved by the Ethics Committee of ISPE Srl (Project identification code 80/17/00)."

\subsubsection{Mathematical Elaboration}

Mean values, standard deviations and variations were calculated for each set of values. Following the results of a normality test (Kolmogorov-Smirnov test) the initial and final data were statistically compared by means of a $t$ test for parametric and dependent groups of data. The differences between the groups of values were considered significant when the probability $p$ was $\leq 0.05$.

\section{Results and Discussion}

\subsection{In Silico}

Molecular dynamics simulations of $1 \mathrm{M}$ aqueous solutions of arginine, taurine and myo-inositol were performed. The co-solvents were present either alone or in mixture with different ratios, but the total osmolytes concentration was always fixed to $1 \mathrm{M}$. The first visual analysis of the trajectories immediately revealed a qualitative difference between the two amino acids and myo-inositol (non-reported). While arginine and taurine during simulation remained uniformly distributed in the volume of the solvation box, myo-inositol tended to rapidly form aggregates. This difference is due to the structure and properties of the different molecules. Arginine, as a charged molecule, does not obviously show any tendency to aggregate, and also taurine, because of its zwitterionic structure, tends to be highly solvated. Myo-inositol instead, being neutral and rich in hydroxyl groups, can readily form a network of hydrogen bond type interactions both with the solvent and with other molecules of myo-inositol. In addition, hydrophobic and Van der Waals interactions between the sugar rings of myo-inositol contribute to make self-aggregation favorable from an entropic and energetic point of view. A subsequent quantitative analysis of the structural and dynamical properties of the mixed solutions was performed through an evaluation of water radial distribution functions and water self-diffusion coefficients. It led to the conclusion that myo-inositol induces the greatest effect on the microscopic structure of water, as shown by the increased magnitude of the first peak of the water-oxygen radial distribution function (Figure 1), revealing a more orderly first solvation shell around water molecules.

On the other hand, arginine has a stronger reducing effect on the coefficient of self-diffusion of water (Figure 2), thus affecting water dynamics and its ability to act as the primary denaturant on proteins possibly present in the solution. 


\section{water oxygen radial distribution} function
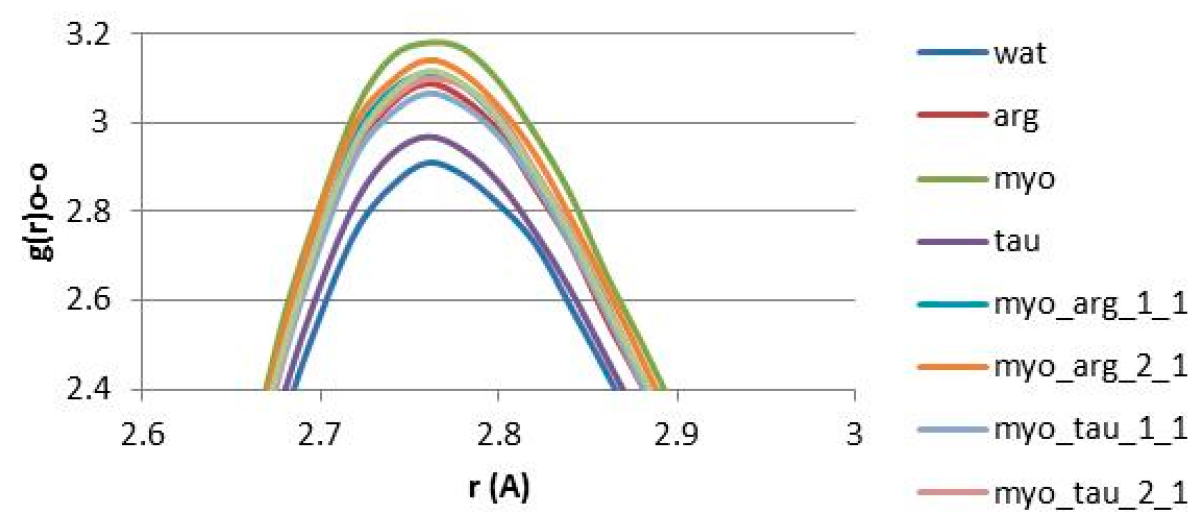

Figure 1. First peak of water-oxygen radial distribution function for pure water and osmolyte solutions.

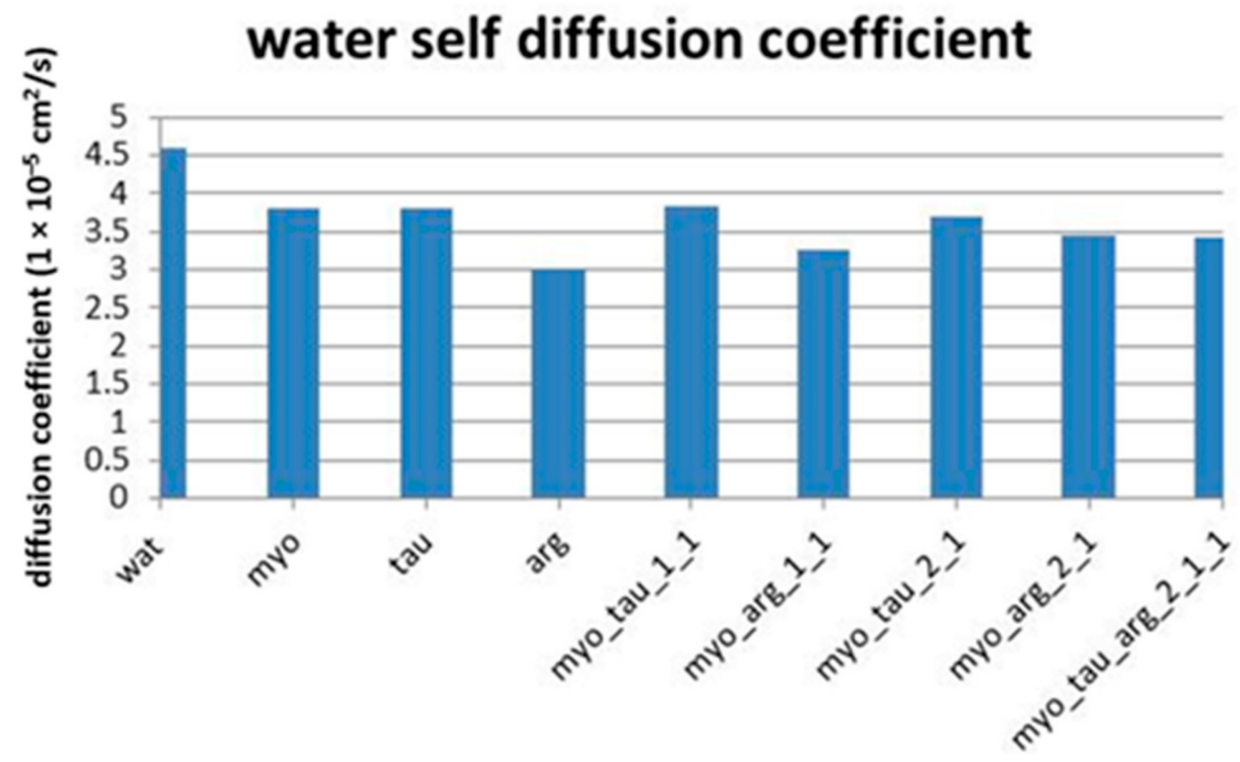

Figure 2. Water self-diffusion coefficient in different solutions as function of the nature and proportion of the co-solvents.

In addition, arginine has a substantial ability to reduce the propensity to cluster shown by myo-inositol. Taurine showed moderate effects both on water dynamics and on water structure. The simulations of co-solvent mixtures in different ratios suggest that the observed effects on the structure and dynamics of water are additive in nature, and their magnitude is proportional to the osmo-protector concentration. This preliminary analysis suggests that a combination of myo-inositol and arginine in 1:1 ratio may be a promising candidate as a blend with osmo-protecting activity, taking advantage of the complementary characteristics of the two molecules.

In the second phase of our study, we investigated the interaction of a keratin model segment with a solutions of the two osmoprotectors found to be most promising in the previous phase, i.e., myo-inositol and arginine. It was possible to observe that myo-inositol had a marked tendency to crowd around keratin, while this trend was not observed for arginine. In the 1:1 mixture, the two osmo-protectors retained their basic behavior, with myo-inositol crowding around keratin, such as to constitute a first osmotic protection layer on the protein, while arginine remained on average more distant, building up a second, more diffused layer (Figure 3) thus reducing the aggregation of myo-inositol, that otherwise would have been more pronounced. 


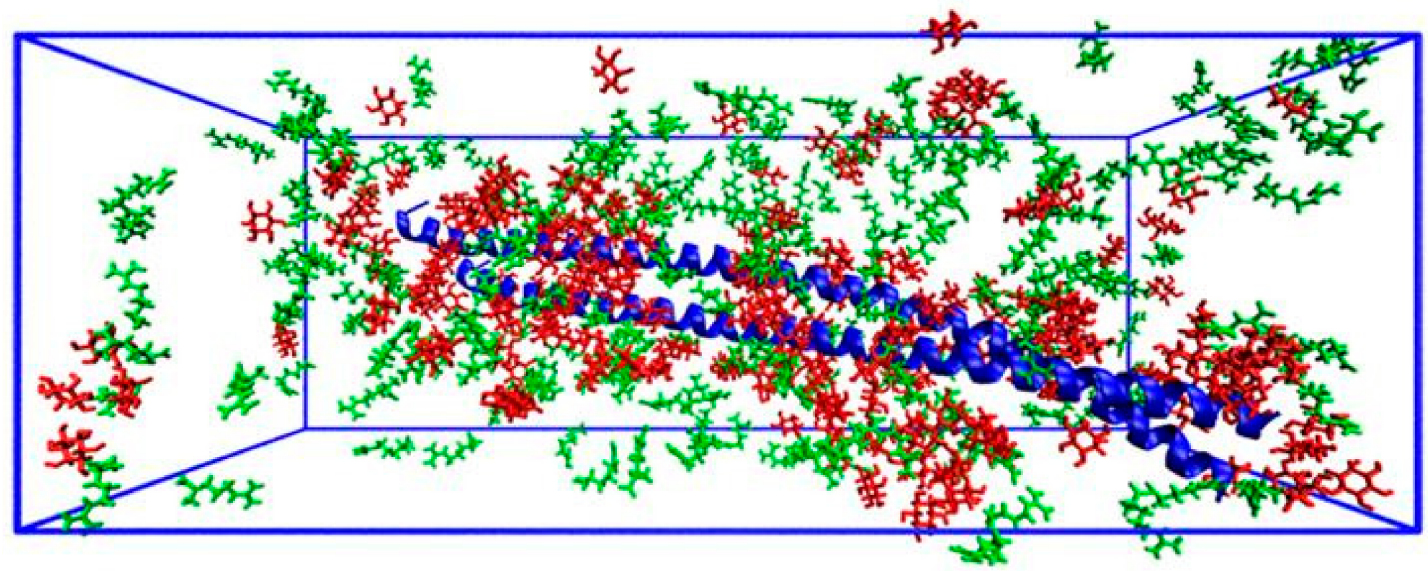

Figure 3. Representative structure of the distribution of osmo-protectors around keratin in a 1:1 mixture containing myo-inositol (red molecules) and arginine (green molecules). Water has been omitted for clarity.

In the arginine-myo-inositol 2:1 mixture, the excess arginine drastically reduced the aggregation of myo-inositol and also significantly reduced its crowding around the protein. In this case, it was not possible to identify any layer of myo-inositol next to the keratin. At a different ratio between the molecules, i.e., with an arginine-myo-inositol 1:2 blend, the behavior with the excess myo-inositol was not substantially changed by arginine, also regarding its aggregation tendency.

These data, according to what was observed in the water-osmoprotectors systems simulations, suggest that the ratio 1:1 is to be preferred for the arginine-myo-inositol combination order to maximize its osmoprotecting potential.

\subsection{In Vitro}

\subsubsection{Histo-Morphological Analysis}

Negative controls: $\mu \mathrm{HF}$ presented a stable morphology, consisting in a compact core formed by dermal papilla fibroblasts surrounded by a multi-layered stratum of hair follicle keratinocytes.

Cyclosporine A did not induce any visible modification of the structure after $24 \mathrm{~h}$, but after $120 \mathrm{~h}$ the nuclei appeared to be denser compared to the surrounding cytoplasm, indicating a possible toxic effect of the compound reference concentration.

Myo-inositol $0.5 \%$ : both after $24 \mathrm{~h}$ and $120 \mathrm{~h}$ treatments, the keratinocyte layers appeared to be more connected to the dermal core and a lower stratification was present, compared to negative control.

Myo-inositol + Arginine mix $(0.25 \%+0.25 \%)$ : after $24 \mathrm{~h}$, the morphology was not different from the Myo-inositol treated sample. On the contrary, at T6 the morphology indicated an increased cells differentiation resulting in a partial detachment of epithelial layers from the dermal core (Figure 4). Keratinocyte layers presented a better organization in lamellar structures and higher thickness compared to the negative control, where epithelial layers were thinner and less compact (higher tissue fragility).

The treatment with the active mix for $120 \mathrm{~h}$ appears to have promoted the keratinocytes differentiation, without inducing tissue fragility and cell loss. 
$H \& E$

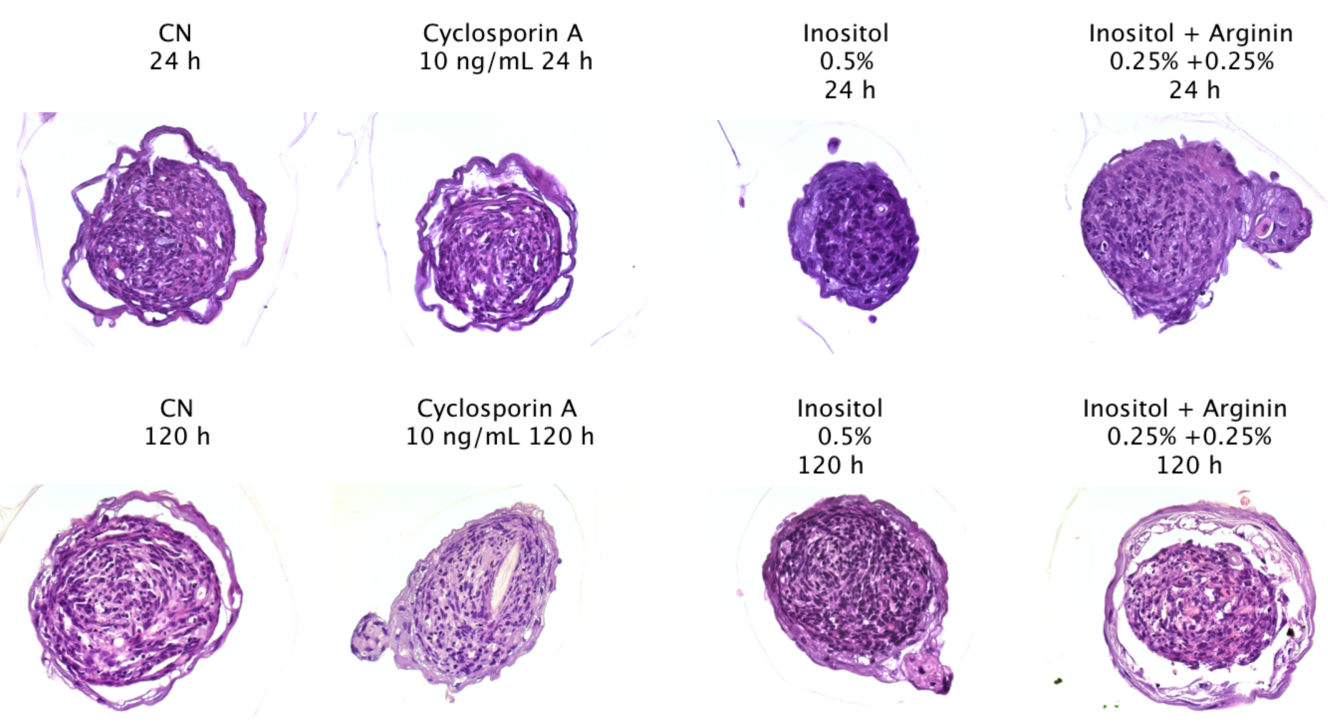

Figure 4. Morphological analysis $\mu \mathrm{HF}$ after 24 and $120 \mathrm{~h}$ of treatment (H\&E staining $40 \times$ magnification).

\subsubsection{Immunostaining}

CK6 is a type II keratin present in the skin and the hair follicle and shows an important role in the intermediate filament formation. Probably, the CK6 protein provides the correct architecture to support cell migration and resistance $[21,22]$ and, as a consequence, to give structure and strength to the skin and its appendages.

Cyclosporine A: In comparison with the negative control, the keratinocyte layers turned out to be thicker and presented a more intense staining, confirming the role of cyclosporine A as active molecule on epithelial compartment.

Myo-inositol $0.5 \%$ : the structure of the $\mu \mathrm{HF}$ and the staining was not significantly different from Cyclosporin A.

Mix Myo-inosotol + Arginine $0.25 \%+0.25 \%$ : in the treated samples, the keratinocyte compartment layer appeared to be more differentiated compared to Cyclosporine A and Myo-inositol alone (the structure appeared thicker and layered). The most relevant result was the strong CK6 signal (Figure 5), corresponding to an overexpression of CK6 compared to all the other samples. This result could indicate a possible effect of the mix Myo-inositol + Arginine $0.25 \%+0.25 \%$ on the terminal differentiation of keratinocytes, which are capable of forming well organized layers and of producing a high quantity of structural keratin CK6.

Collagen IV (Figure 6) is the major component of basal lamina and is part of the follicular dermal-epidermal junction, but is also present in granular extracellular form within the follicular sheath, where it exerts an anchor function during the active growth (anagen) phase of the hair follicle cycle [23]. 
CK6
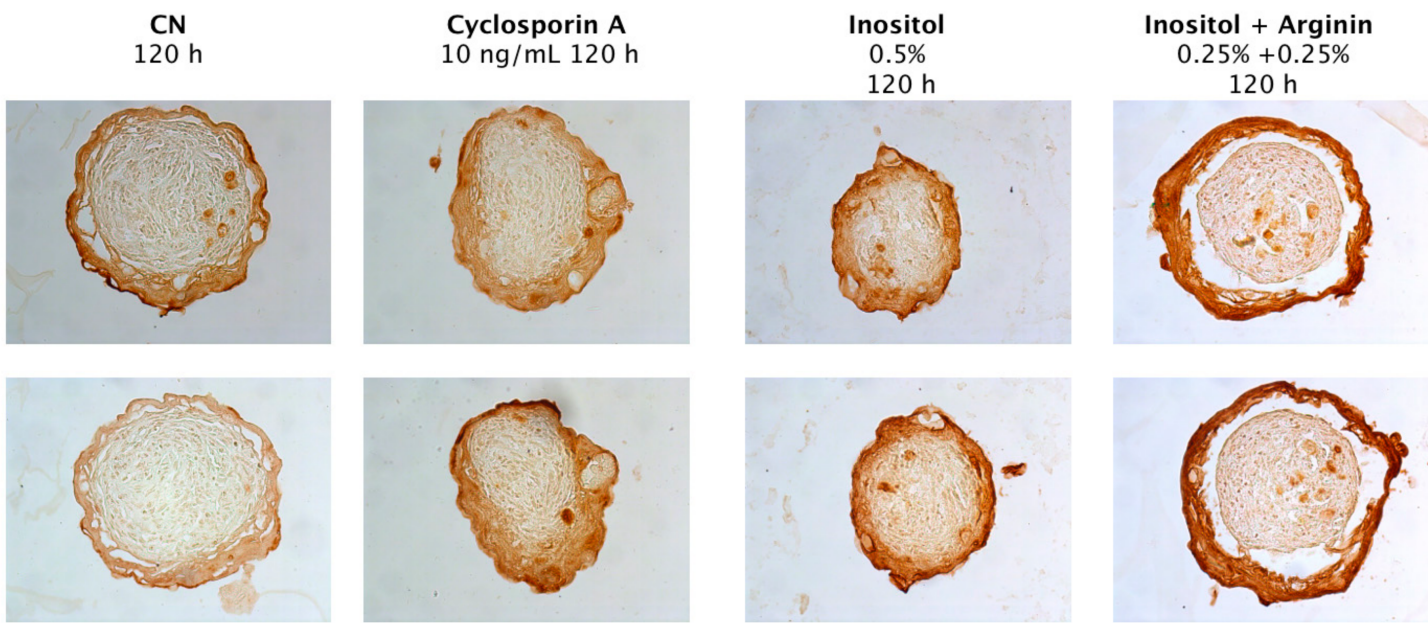

Figure 5. Immunostaining of $\mathrm{CK} 6$. $\mathrm{CN}$ = negative control. $\mathrm{CK} 6$ immuno-stained with specific antibody and marked with DAB (Brown) Magnification $40 \times(\mathrm{n}=2$; biological duplicate except Cyclosporin A $\mathrm{n}=1)$.

\section{COLIV}
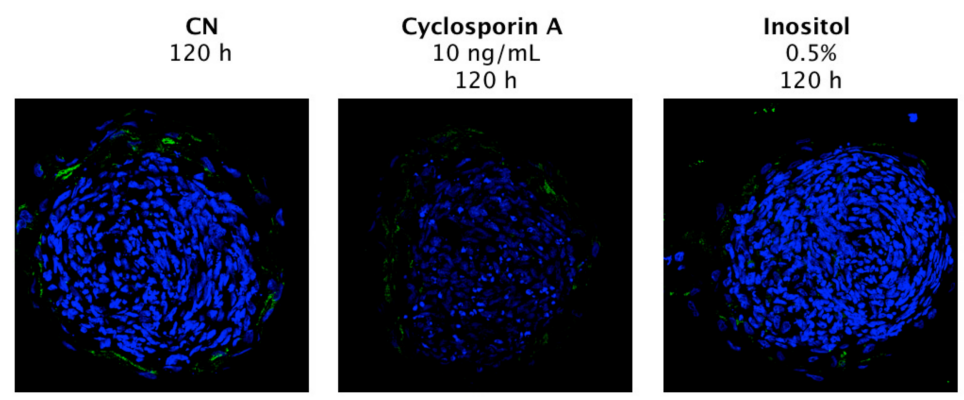

Inositol + Arginin

$0.25 \%+0.25 \%$

$120 \mathrm{~h}$
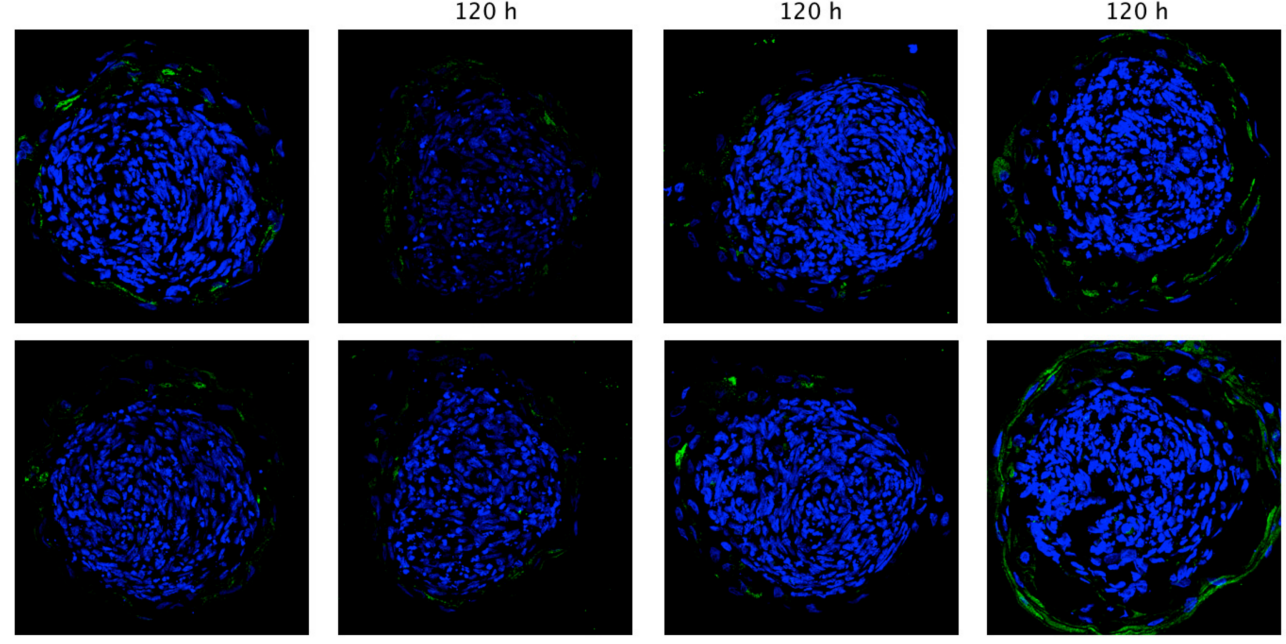

Figure 6. Expression of COLIV CN = negative control. COLIV immunostaining with specific antibody in green (DAPI, blue, nuclei) Magnification $63 \times$. $(n=2$; biological duplicate).

Cyclosporine A, Myo-inositol 0.5\%: the COLIV immun-staining did not show visible differences compared to negative control.

Mix Myo-inosotol + Arginine $0.25 \%+0.25 \%$ : in the treated samples, the expression of the protein IV was significantly increased by the application of active Mix Inositol + Arginine $0.25 \%+0.25 \%$ : for $120 \mathrm{~h}$, in particular at the borders of the core, near the keratinocytes.

The application of mix Myo-inositol + Arginine $0.25 \%+0.25 \%$ induced an increase of the COLIV protein expression, indicating a role in the production enhancement of structural protein of the basal lamina and, as a consequence, of the firmness and strength of the hair follicle. 


\subsection{Ex Vivo}

Determination of washing procedure and associated mass losses.

The first step was to optimize a system to remove the HSP, and to control the hair mass loss associated with the cleaning step. Water, $2 \%$ SDS and $2 \%$ of commercial shampoo were assayed. The procedure that consists in 4 washes exchanging $1 \mathrm{~mL}$ of clean $2 \%$ SLS after a soft shake was considered the best option: It produced less mass loss and removed the HSP. The coefficients of mass loss after the washing step were calculated for each sample (Figure 7A,B), and were used for the final mass correction.
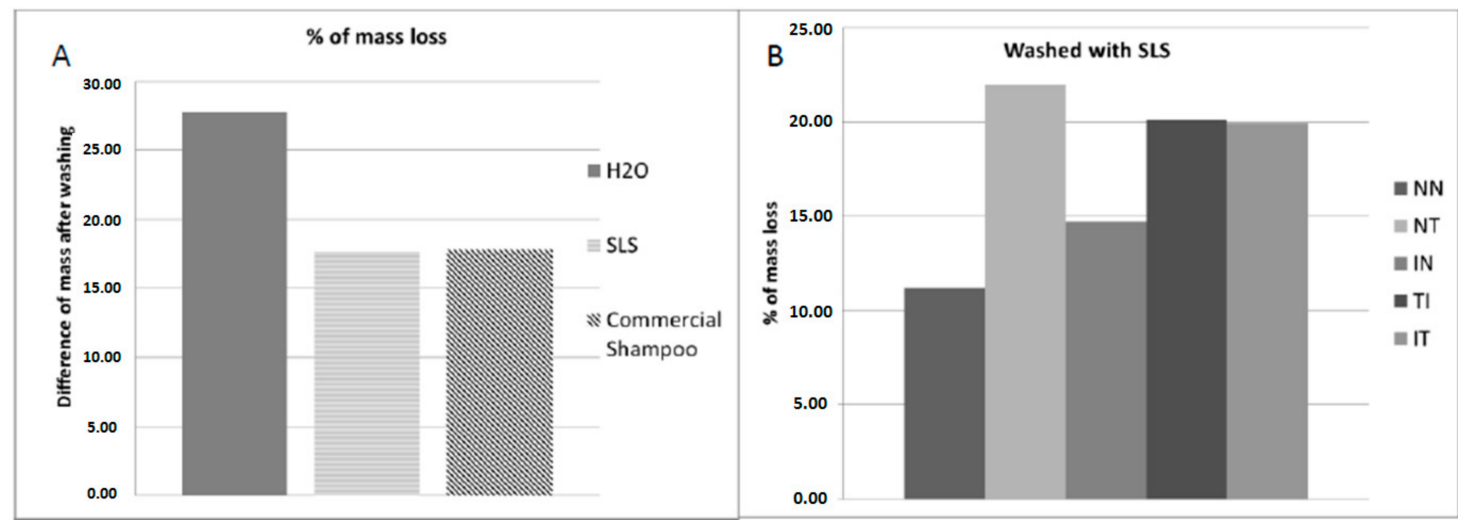

Figure 7. Determination of mass losses associated to washing step. (A) Three different solutions were tested $\left(\mathrm{H}_{2} \mathrm{O}\right.$; sodium lauryl sulphate; commercial shampoo) and the average mass loss was calculated for each one; (B) Mass loss with SLS washing procedure was calculated for each sample. NN: non-irradiated \& non-treated. NT: non-irradiated \& treated. IN: irradiated \& non-treated. TI: treated and irradiated $1 \mathrm{~h}$ later. IT: treated after irradiation.

Protein Degradation Analysis

Following the procedure described in the experimental part, three Bradford analysis of the protein degradation were performed. With the BSA standard the amount of protein solubilized in each sample, directly correlated with the level of protein degradation, could be analyzed. All the data of each experiment are shown in Figure 8. Using the Bradford results and taking into account the different mass loss of each sample, the increase or decrease of the protein degradation was calculated with respect to the NN sample (non-irradiated and non-treated).

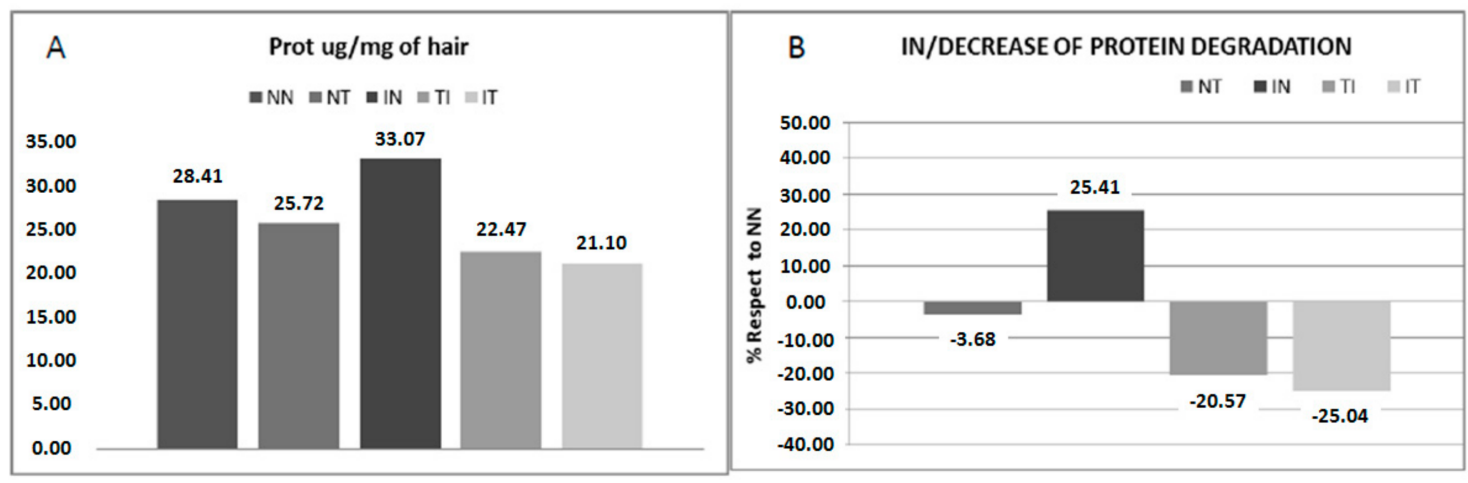

Figure 8. Bradford analysis of protein degradation. Three Bradford analyses of the protein degradation were performed. (A) The mean of the protein concentrations for each sample; (B) The representation of the differences between NN and the other samples in \%. Positive numbers indicate an increased protein degradation while a negative number indicates its reduction. NN: non-irradiated \& non-treated. NT: non-irradiated \& treated. IN: irradiated \& non-treated. TI: treated and irradiated $1 \mathrm{~h}$ later. IT: treated after irradiation. 
The results show that when the hair was irradiated without any treatment, the protein degradation increased $(+25.41 \%)$. In contrast, in all the other groups, with HSP treatment, the protein degradation was reduced, even though at different intensities. The NT group showed a smooth decrement in degradation $(-3.68 \%)$, and the TI and IT groups showed stronger decreases $(-20.57 \%$ and $-25.04 \%$ respectively). The differences between IN and TI vs. NN and between NT, TI, IT vs. IN were significant. The results confirm that the irradiation increases the protein degradation, and the treatment with HSP $1 \mathrm{~h}$ before the exposure not only protects the hair but also decreases the level of protein degradation compared to NN (non-irradiated and non-treated).

\subsection{In Vivo}

After 6 weeks of use of the hair-care treatment including shampoo, spray, serum and hair conditioner, the pull test showed a significant reinforcing effect $(p<0.05)$ with $67.1 \%$ increase in hair resistance to the pulling action, a lower number of removed hairs and higher the hair resistance to traction (Figure 9). A potential increase in the hair diameter, equal to $5.1 \%$ was also evident at the end of the treatment (Figure 10). This was also proved by the positive subjective assessments concerning the perceived efficacy (Figure 11).

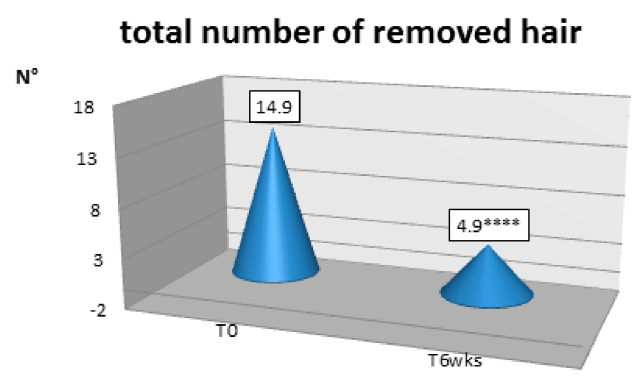

Figure 9. Pull test $\left.{ }^{* * * *} p<0.0001\right)$.

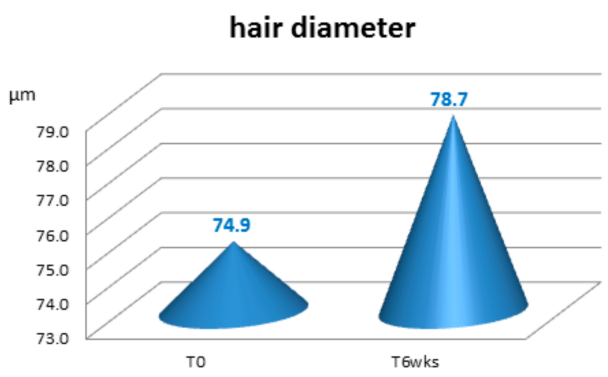

Figure 10. Hair diameter.

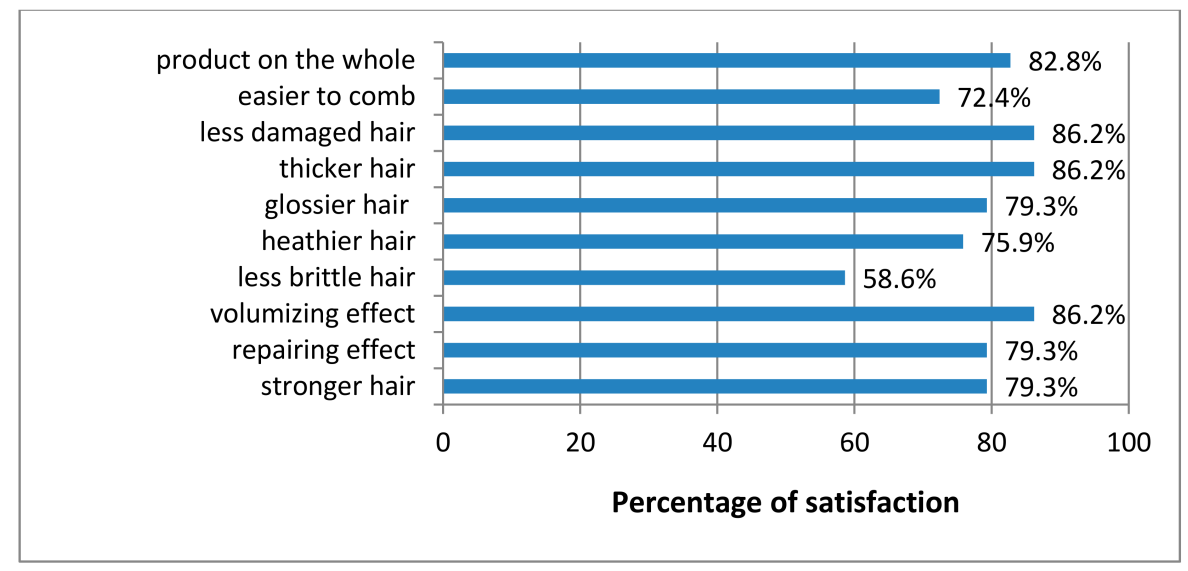

Figure 11. Subjective evaluation. 


\section{Conclusions}

The computerized models adopted allowed us to understand that the ratio 1:1 arginine-myo-inositol is preferable to maximize the osmo-protecting efficacy with keratin protein.

The in vitro model confirmed that the synergic efficacy of the two molecules, myo-Inositol + Arginine, induces peculiar effects of morphology and structural features of $\mu \mathrm{HF}$ compared to myo-inositol alone and Cyclosporine A (reference for hair growth). At a morphological level, this combination seems to promote the keratinocytes differentiation without inducing tissue fragility and cell loss. At the protein expression level, the studied blend noticeably increases the expression of CK6. Moreover, it maintains the morphology of the keratinocytes strata, which keeps it similar to the negative control.

The collagen IV signal is strongly expressed in the keratinocytes strata in the mix-treated samples compared to negative control and the myo-inositol alone. This suggests an enhancement of the production of structural proteins of the basal lamina.

The above results were significantly confirmed by the application of finished products on hair tresses and in the in vivo tests. In particular, the ex vivo hair study clearly indicates that the treatment with the hair spray reduces the effects of UV irradiation, diminishing the protein degradation induced by UV exposure. Some protection effect is found also on virgin hair. However, the effect of the spray is stronger with UV damaged hair, inducing an important, significant reduction of degradation. Same statistically significant results were obtained with volunteer experiments, in terms of increasing the hair strength.

Author Contributions: Conceptualization, G.B., L.R.; Methodology, G.B.; Software, M.S., S.P.; Validation, G.B.; Investigation, E.C., M.M., A.B., M.S., S.P., M.O., L.C.; Data Curation, N.L.; Writing-Original Draft Preparation, N.L.; Writing-Review \& Editing, L.R., N.L.; Supervision, G.B., L.R.

Conflicts of Interest: The authors declare no conflict of interest.

\section{References}

1. Chuong, C.M.; Landes, R.G. Molecular Basis of Epithelial Appendage Morphogenesis; Landes Bioscience: Austin, TX, USA, 1998.

2. Hardy, M.H. The secret life of the hair follicle. Trends Genet. 1992, 8, 55-61. [CrossRef]

3. Cotsarelis, G.; Sun, T.T.; Lavker, R.M. Label-retaining cells reside in the bulge area of pilosebaceous unit: Implications for follicular stem cells, hair cycle, and skin carcinogenesis. Cell 1990, 61, 1329-1337. [CrossRef]

4. Lyle, S.; Christofidou-Solomidou, M.; Liu, Y.; Elder, D.E.; Albelda, S.; Cotsarelis, G. The C8/144B monoclonal antibody recognizes cytokeratin 15 and defines the location of human hair follicle stem cells. J. Cell Sci. 1998, 111, 3179-3188. [PubMed]

5. Paus, R.; Cotsarelis, G. The biology of hair follicles. N. Engl. J. Med. 1999, 341, 491-497. [CrossRef] [PubMed]

6. Rendl, M.; Lewis, L.; Fuchs, E. Molecular dissection of mesenchymalepithelial Interactions in the hair follicle. PLoS Biol. 2005, 3, 331. [CrossRef] [PubMed]

7. Higginsa, C.A.; Chen, J.C.; Cerise, J.E.; Jahoda, C.A.B.; Christiano, A.M. Microenvironmental reprogramming by threedimensional culture enables dermal papilla cells to induce de novo human hair-follicle growth. Proc. Natl. Acad. Sci. USA 2013, 110, 19679-19688. [CrossRef] [PubMed]

8. Lee, C.H.; Kim, M.S.; Chung, B.M.; Leahy, D.J.; Coulombe, P.A. Structural basis for heteromeric assembly and perinuclear organization of keratin filaments. Nat. Struct. Mol. Biol. 2012, 19, 707-715. [CrossRef] [PubMed]

9. Jorgensen, W.L.; Chandrasekhar, J.; Madura, J.D.; Impey, R.W.; Klein, M.L. Comparison of simple potential functions for simulating liquid water. J. Chem. Phys. 1983, 79, 926-935. [CrossRef]

10. Darden, T.; York, D.; Pedersen, L. Particle mesh Ewald: An Nlog(N) method for Ewald sums in large systems. J. Chem. Phys. 1993, 98, 10089-10092. [CrossRef]

11. Pronk, S.; Páll, S.; Schulz, R.; Larsson, P.; Bjelkmar, P.; Apostolov, R.; Shirts, M.R.; Smith, J.C.; Kasson, P.M.; Van der Spoel, D.; et al. GROMACS 4.5: A high-throughput and highly parallel open source molecular simulation toolkit. Bioinformatics 2013, 29, 845-854. [CrossRef] [PubMed] 
12. Bussi, G.; Donadio, D.; Parrinello, M. Canonical sampling through velocity rescaling. J. Chem. Phys. 2007, 126, 014101. [CrossRef] [PubMed]

13. Berendsen, H.J.C.; Postma, J.P.M.; van Gunsteren, W.F.; DiNola, A.; Haak, J.R. Molecular dynamics with coupling to an external bath. J. Chem. Phys. 1984, 81, 3684-3690. [CrossRef]

14. Hess, B.; Bekker, H.; Berendsen, H.J.C.; Fraaje, J.G.E.M. LINCS: A Linear Constraint Solver for Molecular Simulations. J. Comput. Chem. 1997, 18, 1463-1472. [CrossRef]

15. Lindorff-Larsen, K.; Piana, S.; Palmo, K.; Maragakis, P.; Klepeis, J.L.; Dror, R.O.; Shaw, D.E. Improved side-chain torsion potentials for the Amber ff99SB protein force field. Proteins 2010, 78, 1950-1958. [CrossRef] [PubMed]

16. Wang, J.; Wolf, R.M.; Caldwell, J.W.; Kollman, P.A.; Case, D.A. Development and testing of a general amber force field. J. Comput. Chem. 2004, 25, 1157-1174. [CrossRef] [PubMed]

17. Guidelines for the Evaluation of the Efficacy of Cosmetic Products-COLIPA Guidelines; The European Coemetics Association: Auderghem, Belgium, 2008.

18. Piérard, G.E.; Piérard-Franchimont, C.; Marks, R.; Elsner, P. EEMCO guidance for the assessment of hair shedding and alopecia. Skin Pharmacol. Physiol. 2004, 17, 98-110. [CrossRef] [PubMed]

19. Pariser, D.M.; Caserio, R.J.; Eaglestein, W.H. Techniques for Diagnosing Skin and Hair Disease; Thieme: New York, NY, USA, 1986.

20. Van Neste, D.; Lachapelle, J.M.; Antoine, J.L. Trends in Human Hair Growth and Alopecia Research; Springer: Berlin, Germany, 1989.

21. Wong, P.; Coulombe, P.A. Loss of keratin 6 (K6) proteins reveals a function for intermediate filaments during wound repair. J. Cell Biol. 2003, 163, 327-337. [CrossRef] [PubMed]

22. Wojcik, S.M.; Longley, M.A.; Roop, D.R. Discovery of a novel murine keratin 6 (K6) isoform explains the absence of hair and nail defects in mice deficient for K6a and K6b. J. Cell Biol. 2001, 154, 619-630. [CrossRef] [PubMed]

23. Jahoda, C.A.; Mauger, A.; Bard, S.; Sengel, P. Changes in fibronectin, laminin and type IV collagen distribution relate to basement membrane restructuring during the rat vibrissa follicle hair growth cycle. J Anat. 1992, 181, 47-60. [PubMed] 